\title{
Spectral element-Fourier methods applied to simulation of turbulent pipe flow
}

\author{
D. M. McIver* \\ H. M. Blackburn ${ }^{\dagger}$ \\ G. J. Nathan*
}

(Received 7 August 2000)

\begin{abstract}
We present results from direct numerical simulations of turbulent pipe flow at a Reynolds number of 5000. The spatial discretisation employs Fourier expansions in one geometrically homogeneous direction coupled with two-dimensional spectral elements in the remaining
\end{abstract}

* Department of Mechanical Engineering, The University of Adelaide, North Terrace, Adelaide 5005, Australia. mailto:dmciver@mecheng.adelaide.edu.au

$\dagger$ CSIRO Building Construction and Engineering, PO Box 56, Highett, VIC 3190, Australia. mailto:hugh.blackburn@dbce.csiro.au

${ }^{0}$ See http://anziamj . austms.org. au/V42/CTAC99/Mciv for this article and ancillary services, (C) Austral. Mathematical Soc. 2000. Published 27 Nov 2000. 
two coordinates. The geometry under study has two geometrically homogeneous directions, axial and azimuthal, and we compare statistics from two sets of simulations that employ Fourier expansions for each of these two directions in turn. For the case with Fourier expansions in the azimuthal direction, a cylindrical coordinate system is used.

\section{Contents}

\section{Introduction}

C956

2 Computational Technique

C957

3 Meshes

C963

3.1 Cartesian coordinate simulation . . . . . . . . . . C964

3.2 Cylindrical coordinate simulation ... . . . . . . C965

4 Results

C966

4.1 Qualitative flow features . . . . . . . . . . . . C966

4.2 Energy spectra . . . . . . . . . . . . . . . C966

4.3 Mean velocity . . . . . . . . . . . . . . C968

4.4 Fluctuating velocities . . . . . . . . . . . . . . C971

4.5 Turbulent shear stress . . . . . . . . . . . . C971

5 Discussion and Conclusion

C973 


\section{Introduction}

Direct numerical simulation (DNS) is a tool that can be used to build a statistical database for turbulent flows. By resolving all dynamically significant scales of the flow a complete picture of the behaviour of the flow is obtained. Flow features can be visualised and turbulence statistics analysed to aid the development of Reynolds-averaged turbulence models, which are widely used in the engineering community. Resolving all relevant scales of the flow makes DNS a tool that, when carefully implemented, is equivalent to a short duration laboratory experiment [4]. The advantage of simulations relative to experiments is that all quantities of interest can be obtained for the whole field, giving a picture of the flow being studied that can only be matched by advanced experimental techniques. The Reynolds number limitations present in DNS studies can to an extent be relaxed by use of large eddy simulation (LES), which employs turbulence models only for the finest length scales in the flow.

This paper presents results from two simulations of turbulent pipe flow, one performed using a Cartesian coordinate formulation, the other using a cylindrical coordinate formulation. Comparing results for these two simulations is of interest to us because we hope to simulate jet-type flows. The geometry of jet-type flows is easily replicated using cylindrical coordinates, 
though this advantage is somewhat offset by added computational difficulties. Before proceeding to the more complicated jet simulations the accuracy of the cylindrical coordinate code must be verified. Turbulent pipe flow provides a means to check the accuracy of the code as both experimental [2] and computational [3] data is available against which to compare the results. The results will also provide a database of turbulent velocity data that can be used as inlet conditions in later simulation of jet-type flows. Initial conditions for DNS are often generated using this method [5].

The Reynolds number for both simulations was $R e=U D / \nu=5000$, where $U$ is the superficial mean flow speed; Reynolds number based on the friction velocity $u_{\tau}=\left(\tau_{w} / \rho\right)^{1 / 2}, R e_{\tau}=u_{\tau} D / \nu=343$. The friction velocity Reynolds number used in previous experimental investigations are $R e_{\tau}=$ $u_{\tau} D / \nu=338$ [2] and for the computations $R e_{\tau}=u_{\tau} D / \nu=360$ [3].

\section{Computational Technique}

The system under study is governed by the incompressible Navier-Stokes equations

$$
\begin{aligned}
\frac{\partial \boldsymbol{u}}{\partial t}+\boldsymbol{N}(\boldsymbol{u}) & =-\nabla P+\nu \nabla^{2} \boldsymbol{u} \\
\nabla \cdot \boldsymbol{u} & =0
\end{aligned}
$$


where $P=p / \rho$ and $\boldsymbol{N}(\boldsymbol{u})$ represents the non-linear terms. In the present formulation, these are implemented in skew-symmetric form, i.e.

$$
\boldsymbol{N}(\boldsymbol{u})=0.5[\boldsymbol{u} \cdot \nabla \boldsymbol{u}+\nabla \cdot \boldsymbol{u} \boldsymbol{u}] .
$$

Two different spatial discretisations have been employed. Both involve a spectral element-Fourier mode formulation, which uses two-dimensional spectral elements in one plane and Fourier expansions for the third (outof-plane) dimension. Using Fourier expansions as the discretisation for the third spatial dimension restricts the possible geometries to those that possess a spatial homogeneity in one direction. The advantage of this formulation lies in the simulation of problems requiring many grid points - analysing the flow using its Fourier components results in a partly decoupled system that is efficient to implement on parallel architecture computers.

The simulation using Fourier expansions in the pipe axis direction will be referred to here as the Cartesian simulation. The simulation that employs Fourier expansions in the azimuthal, or circumferential, direction in conjunction with a cylindrical coordinate system will be referred to as the cylindrical simulation.

For the Cartesian coordinate system, the introduction of Fourier expansions in the $z$ coordinate direction leads to

$$
\boldsymbol{u}(x, y, z)=\sum_{k}\left\{\begin{array}{c}
\hat{u}_{k}(x, y) \\
\hat{v}_{k}(x, y) \\
\hat{w}_{k}(x, y)
\end{array}\right\} e^{i \beta k z}
$$


with $\beta=2 \pi / L_{z}$, where $L_{z}$ is the domain length in the homogeneous $(z)$ direction and $k$ is a Fourier mode index. As a consequence,

$$
\frac{\partial \boldsymbol{u}}{\partial z}=\sum_{k} \hat{\boldsymbol{u}}_{k} i \beta k e^{i \beta k z} ; \quad \frac{\partial^{2} \boldsymbol{u}}{\partial z^{2}}=\sum_{k}\left(\hat{\boldsymbol{u}}_{k}-\beta^{2} k^{2}\right) e^{i \beta k z} .
$$

By linearity we have

$$
\begin{aligned}
\nabla u= & \sum_{k}\left(\frac{\partial}{\partial x}+\frac{\partial}{\partial y}+i k \beta\right) \hat{u}_{k} e^{i \beta k z} \equiv \sum_{k} \tilde{\nabla} \hat{u}_{k} e^{i \beta k z} \\
\nabla^{2} u & =\sum_{k}\left(\frac{\partial^{2}}{\partial x^{2}}+\frac{\partial^{2}}{\partial y^{2}}-k^{2} \beta^{2}\right) \hat{u}_{k} e^{i \beta k z} \\
& \equiv \sum_{k}\left(\nabla_{x y}^{2}-k^{2} \beta^{2}\right) \hat{u}_{k} e^{i \beta k z} \equiv \sum_{k} \tilde{\nabla}^{2} \hat{u}_{k} e^{i \beta k z}
\end{aligned}
$$

Thus the transformed Cartesian-coordinate Navier-Stokes equations for each mode $k$ are

$$
\begin{aligned}
\frac{\partial \hat{\boldsymbol{u}}_{k}}{\partial t}+\widehat{\boldsymbol{N}(\boldsymbol{u})_{k}} & =-\tilde{\nabla} \hat{P}_{k}+\nu \tilde{\nabla}^{2} \hat{\boldsymbol{u}}_{k}, \\
\tilde{\nabla} \cdot \hat{\boldsymbol{u}}_{k} & =0
\end{aligned}
$$

where $\widehat{\boldsymbol{N}(\boldsymbol{u})_{k}}$ represents the Fourier transform of the nonlinear product terms, which are computed in physical space to avoid formation of convolution sums. In a distributed memory environment where each process holds a 
restricted set of Fourier modes, memory exchanges are required to compute these nonlinear products.

For a cylindrical $(z, r, \theta)$ coordinate system, we introduce $u_{z} \leftrightarrow u, u_{r} \leftrightarrow v$, $u_{\theta} \leftrightarrow w$, and Fourier expansions in the $\theta$ direction. Radial and azimuthal velocities are linearly coupled through the Laplacian:

$$
\tilde{\nabla}^{2}=\frac{\partial^{2}}{\partial z^{2}}+\frac{1}{r} \frac{\partial}{\partial r}\left(r \frac{\partial}{\partial r}\right)+\frac{1}{r^{2}} \frac{\partial}{\partial \theta^{2}} \equiv \nabla_{r z}^{2}-\frac{\beta^{2} k^{2}}{r^{2}} .
$$

This produces linear coupling between the $r$ and $\theta$ components of the transformed Navier-Stokes equations:

$$
\begin{aligned}
\left.\frac{\partial \hat{u}_{k}}{\partial t}+\widehat{\boldsymbol{N}(\boldsymbol{u}}\right)_{z k} & =-\frac{\partial \hat{P}_{k}}{\partial z}+\nu \tilde{\nabla}^{2} \hat{u}_{k} \\
\left.\frac{\partial \hat{v}_{k}}{\partial t}+\widehat{\boldsymbol{N}(\boldsymbol{u}}\right)_{r k} & =-\frac{\partial \hat{P}_{k}}{\partial r}+\nu\left(\tilde{\nabla}^{2} \hat{v}_{k}-\frac{\hat{v}_{k}}{r^{2}}-2 \frac{i \beta k}{r^{2}} \hat{w}_{k}\right) \\
\left.\frac{\partial \hat{w}_{k}}{\partial t}+\widehat{\boldsymbol{N}(\boldsymbol{u}}\right)_{\theta k} & =-\frac{i k \hat{P}_{k}}{r}+\nu\left(\tilde{\nabla}^{2} \hat{w}_{k}-\frac{\hat{w}_{k}}{r^{2}}+2 \frac{i \beta k}{r^{2}} \hat{v}_{k}\right)
\end{aligned}
$$

The change of variables

$$
\tilde{v}_{k}=\hat{v}_{k}+i \hat{w}_{k}, \quad \tilde{w}_{k}=\hat{v}_{k}-i \hat{w}_{k}
$$

is used to decouple the equations for $\hat{v}_{k}$ and $\hat{w}_{k}$ [10], resulting in:

$$
\frac{\partial \hat{u}_{k}}{\partial t}+\widehat{\boldsymbol{N}(\boldsymbol{u})_{z k}}=-\frac{\partial \hat{P}_{k}}{\partial z}+\nu\left[\tilde{\nabla}_{r z}^{2}-\frac{(\beta k)^{2}}{r^{2}}\right] \hat{u}_{k}
$$




$$
\begin{aligned}
\frac{\partial \tilde{v}_{k}}{\partial t}+\tilde{\boldsymbol{N}(\boldsymbol{u})_{r k}} & =-\left[\frac{\partial}{\partial r}-\frac{\beta k}{r}\right] \hat{P}_{k}+\nu\left[\tilde{\nabla}_{r z}^{2}-\frac{(\beta k+1)^{2}}{r^{2}}\right] \tilde{v}_{k}, \\
\frac{\partial \tilde{w}_{k}}{\partial t}+\hat{\boldsymbol{N}(\boldsymbol{u})_{\theta k}} & =-\left[\frac{\partial}{\partial r}+\frac{\beta k}{r}\right] \hat{P}_{k}+\nu\left[\tilde{\nabla}_{r z}^{2}-\frac{(\beta k-1)^{2}}{r^{2}}\right] \tilde{w}_{k},
\end{aligned}
$$

where

$$
\widetilde{\boldsymbol{N}(\boldsymbol{u})_{r k}}=\widehat{\boldsymbol{N}(\boldsymbol{u})_{r k}}+i \widehat{\boldsymbol{N}(\boldsymbol{u})_{\theta k}}, \quad \widetilde{\boldsymbol{N}(\boldsymbol{u})_{\theta k}}=\widehat{\boldsymbol{N}(\boldsymbol{u})_{r k}}-i \widehat{\boldsymbol{N}(\boldsymbol{u})_{\theta k}} .
$$

At each time-step, this change of variables is applied and inverted before and after the viscous sub-step is taken in the operator-splitting scheme used to integrate the Navier-Stokes equations.

It was found that azimuthal low-pass filtering of near-axis velocity values [6] contributed significantly to the numerical stability of the cylindrical formulation. This modification has been recently employed in the simulation of flow past spheroids [8]. Figure 1 shows the form of the filter mask that was applied to the velocity values at the end of each time-step. In effect, the low-pass filtering ensures that nonlinear product terms are fully de-aliased in the azimuthal direction near the axis in the cylindrical formulation.

No explicit de-aliasing is carried out during the formation of the nonlinear product terms $\widehat{\boldsymbol{N}(\boldsymbol{u})_{k}}$ in the Cartesian formulation, although it has been shown [11] that the skew-symmetric form of the nonlinear terms (3) acts to reduce aliasing errors. This reduction (through implicit cancellation of error terms of opposite sign) acts only to reduce aliasing errors in the non-Fourier directions in the present formulation. 


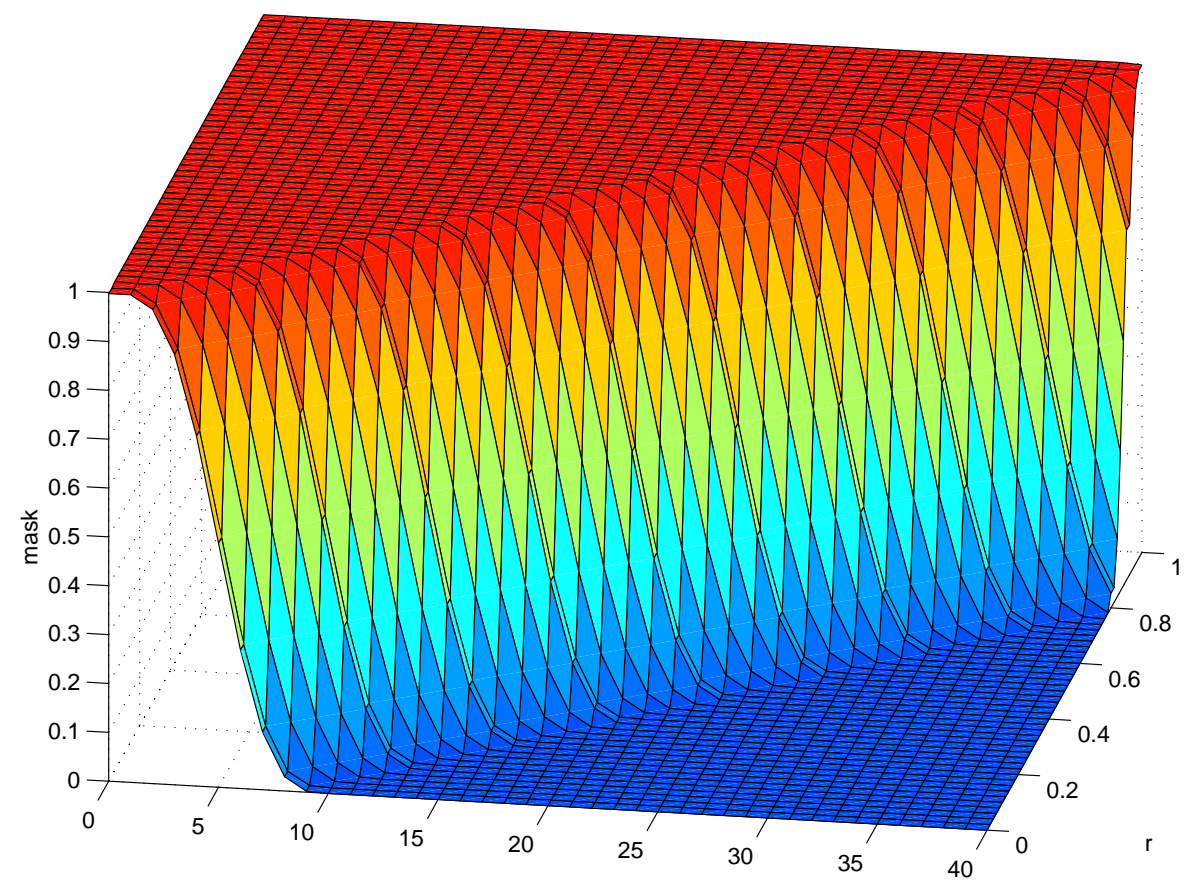

$\mathrm{k}$

Figure 1: Low-pass filter mask applied to Fourier representation of velocity fields in cylindrical formulation. $k$ : Fourier mode number, $r$ : radius $/ r_{\max }$. 
The remainder of the spatial and temporal discretisation is carried out using standard spectral element methods [7].

The flow was driven along the pipe axis by a body force per unit mass of magnitude $4 \tau_{w} / \rho D$ applied to (1). The wall shear stress, $\tau_{w}$, was calculated using the Blasius relationship to allow the body force to be preset. Turbulent flow was initiated from an initial $1 / 7$ power-law profile with superimposed random Gaussian noise at a magnitude of $0.0001 \%$ of the superficial mean velocity $U$. Simulations were run until the measured average wall shear stresses had stabilised near the pre-calculated value, at which point temporal averaging for mean velocities and Reynolds stresses was initiated.

\section{Meshes}

The size of a computational domain must be chosen on the basis that it is sufficiently large to allow the predominant flow structures to develop. A domain length of $L=5 D$ was chosen on the basis that turbulence quantities have been found to be substantially uncorrelated at half this length [3].

Another important consideration is the resolution near the wall. For DNS, the first point away from the wall should be located at $y^{+}<1$, with streamwise spacing $\Delta x^{+} \simeq 6$ and cross flow resolution $\Delta z^{+} \simeq 15$, with scaling in 'wall units', e.g. $x^{+}=x u_{\tau} / \nu[9]$. 


\subsection{Cartesian coordinate simulation}

Spectral element meshes for both simulations are shown in figure 2. The mesh used for the Cartesian simulation has 64 elements, each with 10th order Gauss-Lobatto-Legendre (GLL) tensor-product shape functions. It uses 192 planes (96 complex Fourier modes) of data, which results in representative grid sizes, $\Delta^{+}=\left[(\Delta x \Delta y \Delta z)^{1 / 3}\right]^{+}$, varying from $\Delta^{+} \simeq 4.27$ at the wall, to $\Delta^{+} \simeq 6.41$ at the centre of the pipe.

The resolution used in the comparison simulation of Eggels et al. [3] was $\Delta^{+} \simeq 4.9$ near the wall and decreased towards the centre of the pipe due to the cylindrical coordinate system used. Hence the mean resolution at the wall in the current simulation is of a similar size. However, it should be noted that the streamwise resolution used in the comparison simulation was $\Delta z^{+} \simeq 7.03$ whereas the resolution here is $\Delta z^{+} \simeq 11.2$. This is due to two factors: the domain length used in this simulation is $L=2 \pi D$, broken into 192 data planes compared with a domain length of $L=5 D$ with 256 grid points along the streamwise axis used in the comparison simulation [3]. It could be expected that a lower resolution could be used in the present simulations without sacrificing accuracy, owing to the use of higherorder spatial discretisations (the comparison simulation used only 2nd-order accurate discretisations). The total number of grid points used in the Cartesian simulation was approximately $1.25 \times 10^{6}$, under half that used in the comparison simulation [3]. 

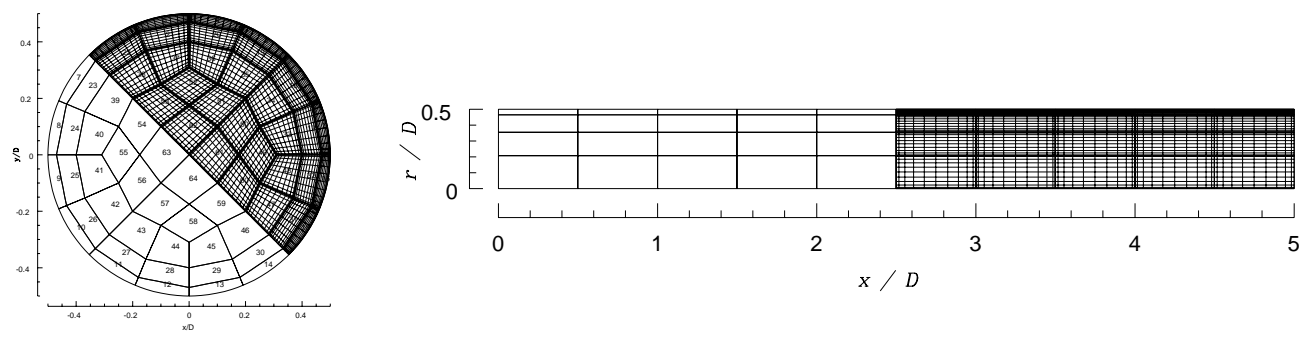

Figure 2: Cartesian and cylindrical spectral element meshes. In each case, internal-element nodes are represented for half the elements.

\subsection{Cylindrical coordinate simulation}

The spatial discretisation for the polar coordinate simulations consisted of the two dimensional mesh of length $L=5 D$ (as shown in figure 2 for the $(r-z)$ plane) combined with 80 planes of data in the azimuthal direction. Streamwise resolution is approximately $\Delta z^{+}=17$, while the approximate wall-normal distance of the first grid-point is $\Delta y^{+}=1.25$. The resulting representative grid size $\Delta^{+}=\left[(\Delta r(r \Delta \theta) \Delta z)^{1 / 3}\right]^{+}$varying from $\Delta^{+} \simeq 6.6$ at the pipe wall to $\Delta^{+} \simeq 5.6$ at the pipe axis. The resolution on the pipe axis in the comparison simulation was $\Delta^{+} \simeq 0.85$ [3], much higher that should be necessary to resolve the flow at the centre line. The number of grid points used in the cylindrical simulation was approximately $3.25 \times 10^{5}$, approximately one quarter that used for the Cartesian simulation, or about one-eighth that used in the comparison simulation. 


\section{Results}

\subsection{Qualitative flow features}

Figure 3 shows contours of instantaneous streamwise velocity near to the wall surface generated from one realisation of the cylindrical simulation. These contours clearly show the unsteadiness of the flow, and the sections of fast and slow moving fluid that make up the near wall flow structures. The periodicity of the domain is also apparent in that the velocity contours at either end of the domain match up.

\subsection{Energy spectra}

The use of Fourier expansions in one coordinate direction simplifies the task of producing one-dimensional energy spectra. We compute the kinetic energy of each mode $k$ as

$$
E_{k}=\frac{1}{2 A} \rho \int_{\Omega} \hat{\boldsymbol{u}}_{k} \cdot \hat{\boldsymbol{u}}_{k}^{*} \mathrm{~d} \Omega,
$$

where $A$ is the area of the two-dimensional domain $\Omega$ and $\hat{\boldsymbol{u}}_{k}^{*}$ is the complex conjugate of velocity field $\hat{\boldsymbol{u}}_{k}$.

Figure 4 shows the ensemble-average energy spectra for the both simulations. In both plots, the dominant energy in the mean flow is reflected by the comparatively high value of $E_{0}$. For the Cartesian simulation, the 


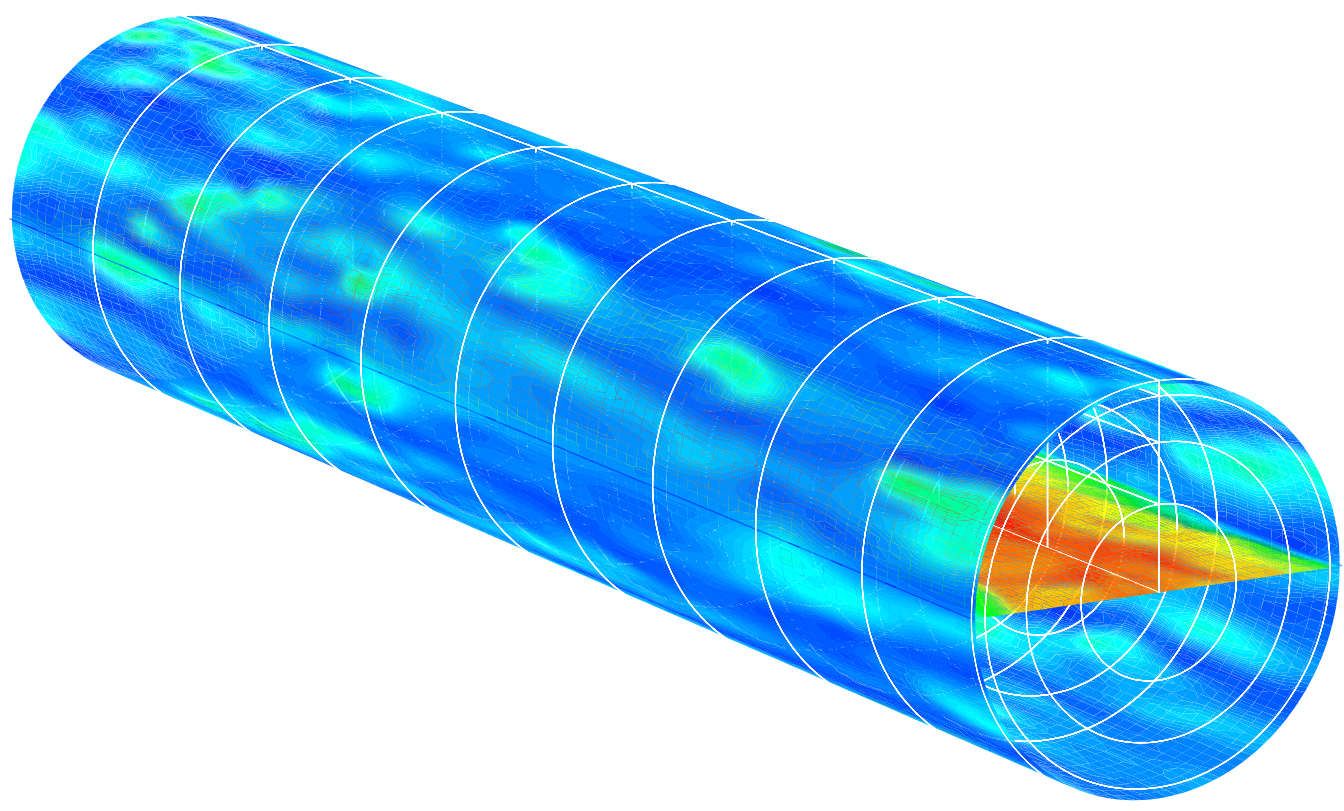

Figure 3: Contours of streamwise velocity on a surface close to the pipe wall and on a plane through the pipe axis. Contours on both surfaces illustrate the near-wall structures of the flow. The outlines of the spectral element mesh for the cylindrical simulation is also shown. 
four decade difference in energy between $E_{1}$ and highest Fourier modes suggests adequate stream-wise resolution. The slight upward trend in the highest modes $(k>80)$ suggests contamination produced by aliasing. For the cylindrical simulation, the roll-off at high wavenumbers is an artefact of the azimuthal filtering discussed in $\S 2$, and it does not appear that aliasing errors are significant. However, the fact that there is only approximately a two-and-a-half decade fall in energy between $E_{1}$ and that for $k \simeq 35$ suggests that the azimuthal resolution is somewhat low.

\subsection{Mean velocity}

Figure $5 a$ ) is a comparison between the mean velocity profiles obtained from the different simulations. The velocity has been non-dimensionalised using the friction velocity, $u_{\tau}$. Experimental data [2] is provided for comparison. It can be seen that the Cartesian simulation provides good agreement with experimental data while the cylindrical simulation differs by approximately $3 \%$ for most of the profile. We attribute the poorer agreement for the cylindrical coordinate simulation to under-resolution - similar effects can be noted in under-resolved DNS data [1]. Figure $5 b$ ) shows the velocity profile re-plotted against distance from the wall (in wall units $u_{\tau} / \nu$ ) along a semi-logarithmic axis. It again shows the significant difference between the experimental data and that from the cylindrical simulation, while the data for the Cartesian simulation are in comparatively good agreement with experimental values. 


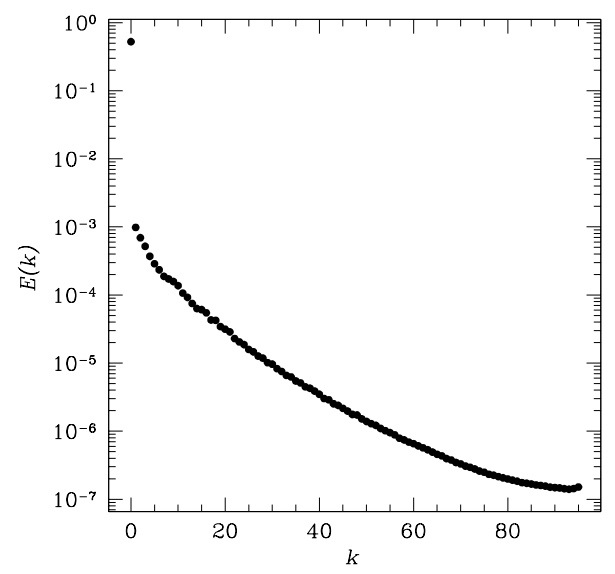

(a)

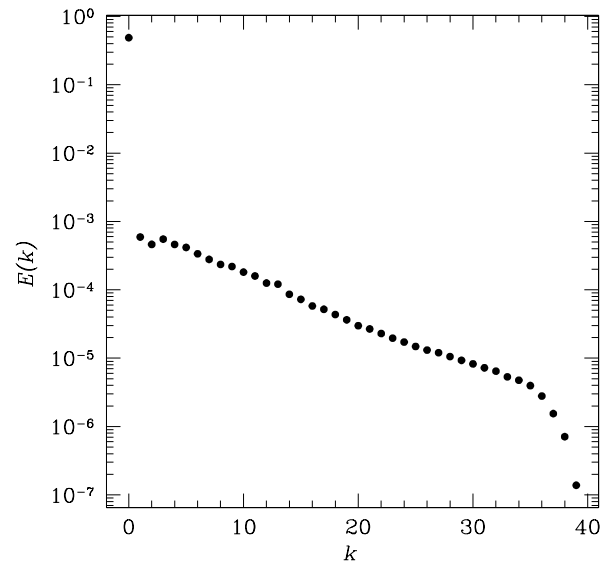

(b)

Figure 4: Ensemble-average energy spectra. The dominance of mode $k=$ 0 in each plot reflects the contribution of the mean stream-wise flow. $a$ ): Cartesian simulation, $b$ ): cylindrical simulation. 


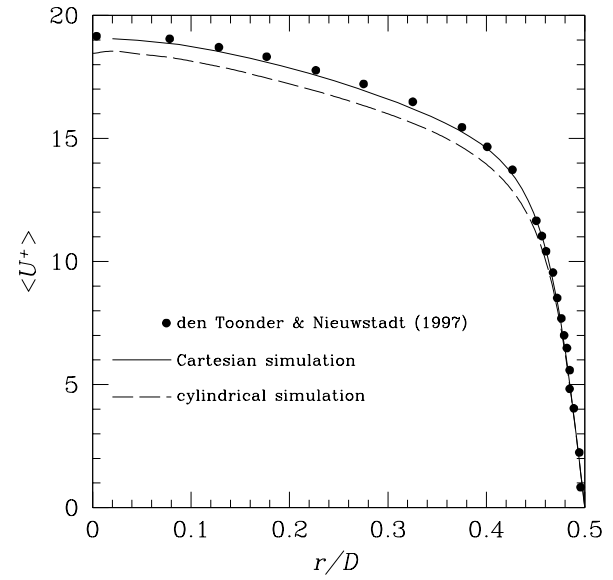

(a)

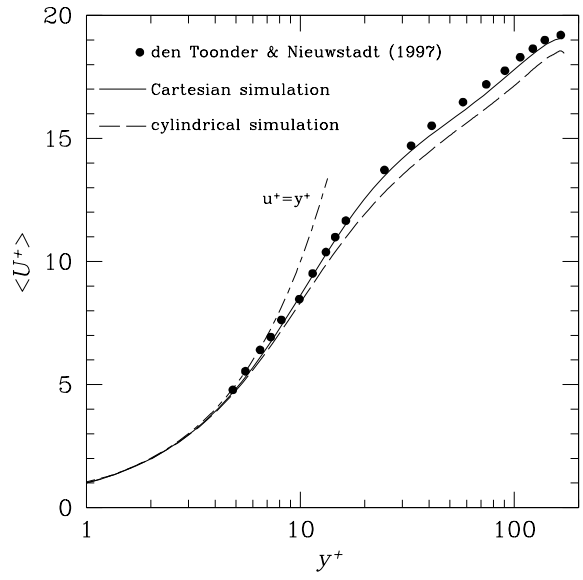

(b)

Figure 5: Non-dimensional velocity profiles from the simulations plotted against $a$ ) pipe radius divided by diameter, and $b$ ) viscous wall units $\nu / u_{\tau}$. Experimental data [2] is shown for comparison. 


\subsection{Fluctuating velocities}

Figure $6 a$ ) shows the fluctuating streamwise standard deviation velocity obtained from the simulations. The results from the Cartesian simulation differ noticeably from the experimental results [2] and simulation results [3]. The results from the cylindrical simulation do not agree well with experimental measurements, another indication of poor spatial resolution.

The results for the fluctuating component of the radial velocity, shown in Figure $6 b$ ) are much closer to the experimental data than was the case for the axial velocity. The results from the Cartesian simulation, especially, provide close agreement to experimental results [2]. The agreement is slightly poorer in the case of the cylindrical simulation, but still quite good, except very close to the pipe axis. For all three simulations, the poor agreement with experimental data very near the wall can be attributed to resolution errors in the experiment.

\subsection{Turbulent shear stress}

The turbulent Reynolds shear stress is plotted as a function of radius in figure 7 . The average total (turbulent + viscous) shear stress gives a basis against which all four sets of results shown can be compared. Again, and in agreement with trends shown in figure 6 , the results from the Cartesian simulation are in reasonable agreement with experimental values, except near 


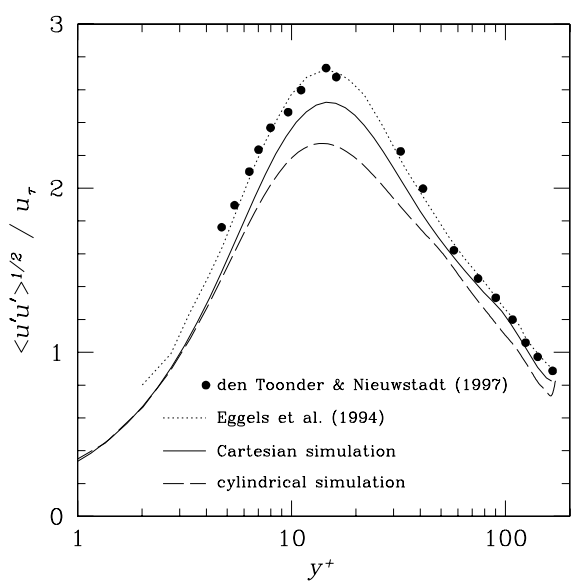

(a)

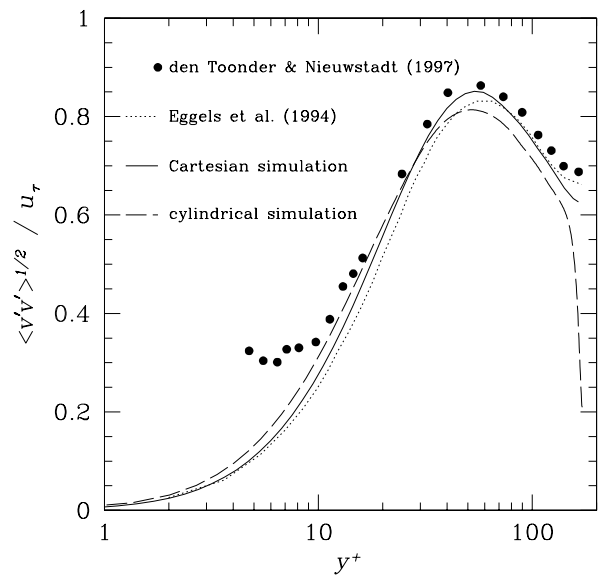

(b)

FiguRE 6: a) Fluctuating streamwise velocity and b) fluctuating radial velocity, both normalised by the friction velocity $u_{\tau}$. Experimental data [2] and simulation results [3] are shown for comparison. 
$r / D=0.4$, while the results from the cylindrical simulation do not agree well with either of the published results.

As shown in Figure 7, results for the Cartesian and the cylindrical simulations agree well with one another in the near-wall region, but less well nearer to the centre of the pipe. This fact, coupled with the lack of smoothness in the profile of turbulent shear stress near the pipe axis, suggests that the lack of spatial resolution in the cylindrical simulation has been most significant in the buffer layer and near the centre of the pipe.

\section{Discussion and Conclusion}

Results from two simulations that employ spectral element-Fourier formulations have been presented. The simulation using the Cartesian coordinate system performed well when compared with experimental data. The Cartesian simulation has provided results that agree well with current experimental and numerical results. We are confident that a database of the instantaneous velocity fields will provide excellent inlet boundary conditions for the simulation of the jet-type flows that will form the main thrust of our future work.

The comparatively poorer performance in the case of the cylindrical coordinate system simulation are provisionally attributed to a lack of spatial resolution. This is based on the fact that the total number of grid points 


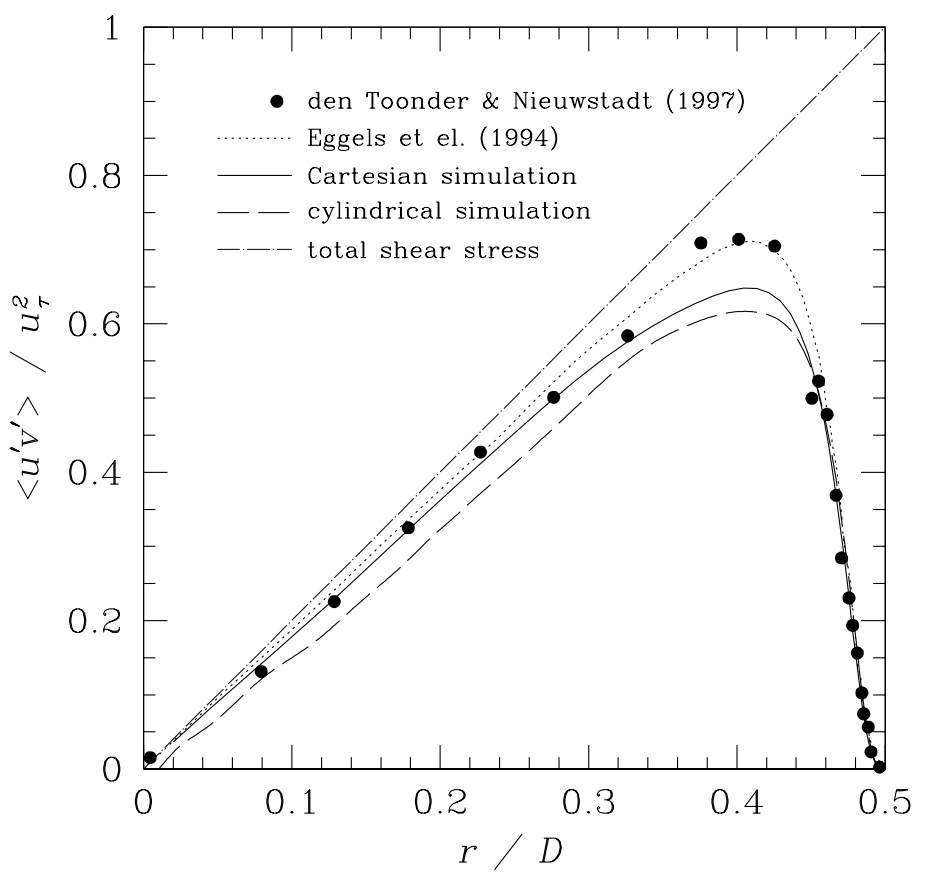

Figure 7: Turbulent Reynolds shear stress. Experimental data [2] and simulation results [3] are shown for comparison. 
is approximately one-eighth that employed in the comparison simulation [3], and approximately one quarter that of the Cartesian simulation. We plan to complete a more detailed study of resolution effects in the near future.

It was found that filtering of velocity data near the axis [6] was required to stabilise the cylindrical simulations, and the effect of the functional form adopted for this filtering needs to be more fully assessed.

Acknowledgements: Computations reported here were carried out at the Australian National University Supercomputing Facility, and we thank the staff of ANUSF for their continuing assistance. Thanks also to Dr Francis Vaughan and the South Australian Centre for Parallel Computing. This work has been supported by Fuel \& Combustion Technologies Pty Ltd.

\section{References}

[1] H. M. Blackburn. Channel flow LES with spectral elements. In M.C. Thompson and K. Hourigan, editors, Thirteenth Australasian Fluid Mechanics Conference, volume 2, pages 989-992, Monash University, Melbourne, Australia, December 1998. C968

[2] J. M. H. den Toonder and F. T. M. Nieuwstadt. Reynolds number effects in a turbulent pipe flow for low to moderate Re. Phys. Fluids, 
9(11):3398-3409, 1997. C957, C957, C968, C970, C971, C971, C972, C974

[3] J. G. M. Eggels, F. Unger, M. H. Weiss, J. Westerweel, R. J. Adrian, R. Friedrich, and F. T. M. Nieuwstadt. Fully developed turbulent pipe flow: a comparison between direct numerical simulation and experiment. J. Fluid Mech., 268:175-209, 1984. C957, C957, C963, C964, C964, C964, C965, C971, C972, C974, C975

[4] Joel H. Ferziger. Simulation and Modelling of Turbulent Flows, chapter 3. Large Eddy Simulation, pages 109-154. Oxford University Press, Inc., 1996. C956

[5] Joel H. Ferziger and Milovan Perić. Computational Methods for Fluid Dynamics. Springer-Verlag, 1996. C957

[6] Bengt Fornberg. A Practical Guide to Pseudospectral Methods. Cambridge University Press, 1996. C961, C975

[7] George Em Karniadakis and Ronald D. Henderson. Spectral element methods for incompressible flows. In R. W. Johnson, editor, Handbook of Fluid Dynamics, chapter 29, pages 29-1-29-41. CRC Press, Boca Raton, 1998. C963

[8] Rajat Mittal. A Fourier-Chebyshev spectral collocation method for simulating flow past spheres and spheriods. Intnl J. Num. Meth. Fluids, 30:921-937, 1999. C961 
[9] Ugo Piomelli. Large-eddy simulations: Where we stand. In C. Liu and Z. Liu, editors, Advances in DNS/LES, pages 93-104, Louisiana, August 1997. AFOSR. C963

[10] A. G. Tomboulides, S. A. Orszag, and G. E. Karniadakis. Direct and large-eddy simulation of axisymmetric wakes. AIAA paper 93-0546, 1993. C960

[11] Thomas A. Zang. On the rotation and skew-symmetric forms for incompressible flow simulations. Appl. Num. Math., 7:27-40, 1991. C961 